\title{
Effect of scattering environment on estimation quality in V2I and V2V communications
}

\author{
Elena Uchiteleva ${ }^{* \dagger}$ and Serguei Primak ${ }^{\dagger}$
}

\begin{abstract}
In this paper, we use a set of modulated discrete prolate spheroidal sequences (MDPSS) to represent a band-limited channel in the scenario with scattering from one or more clusters which can be used in both vehicle-to-infrastructure (V2I) or vehicle-to-vehicle (V2V) communication cases. Then we evaluate the performance of $2 \times 1$ space-time transmit diversity (STTD) system with Alamouti coding and imperfect channel estimation at the receiver. We consider examples of different scattering environments which represent vehicular communication in urban areas, derive expressions for autocorrelation function of channel gains and verify it by simulation. Scattering effect on estimation quality of the system is examined in terms of minimum mean square error (MMSE) and bit error rate (BER).
\end{abstract}

Keywords: Alamouti coding; MIMO channels; Scattering; Channel estimation; DPSS; Wiener filter; STBC; STTD

\section{Introduction}

High-quality channel state information (CSI) is essential for reliable performance of any practical communication system. The most popular approach is estimation via training sequences (pilots) which are periodically inserted into the data stream [1-3]. The receiver extracts pilot sequences and, relying on the knowledge of channel statistics, performs the estimation. For simulation purposes, Rayleigh fading channels with Jake's spectrum [4] and real-valued auto-covariance function are usually assumed, for example in works $[1-3,5,6]$. This is the worst case scenario, since there is no preferable angle of arrival (AoA). Therefore, it leads to unnecessarily large amount of pilots needed for reliable estimation, which is inefficient. Moreover, practical channels usually exhibit non-symmetric spectrum and complex-valued auto-covariance functions. This work is focused on the estimation in a realistic urban environment. Hence, our goal is to account for a complex scenario, which describes scattering from one or more narrow clusters near the mobile, what results in the presence of diffusive components in received signal coming from particular AoA, and to provide qualitative analysis of estimation in different real-life scattering scenarios.

\footnotetext{
*Correspondence: euchitel@alumni.uwo.ca

${ }^{\dagger}$ Equal contributors

Department of Electrical and Computer Engineering, UWO, 1151 Richmond

St., London, N6A 5B9, Canada
}

Measurements show, that realistic spectrum could be represented as a sum of sub channels with a narrow and rectangular spectra $[7,8]$. Therefore, we can assume that the signal spectrum can be approximated as a group of distinct rectangles (corresponding to different clusters) and not as a classical Jake's bathtub shape. Such representation allows us to perform more practical analysis of communication link and obtain more sensible results of estimation quality.

Channel basis expansion models (BEM) recently gained attention due to simplicity of their implementation [6]. For example, some models describing Jake's spectrum include complex-exponential BEM [9] or polynomial BEM [10]. Discrete Karhunen-Loeve BEM is optimal in mean square error (MSE) sense [11,12]. In this expansion, optimal set of basis functions depends on the spectrum shape. It was shown in $[11,13]$ that for a rectangular-shaped spectrum, a set of discrete prolate spheroidal sequences (DPSS) is optimal. Moreover, with the assumption that the spectrum can be approximated as an aggregate of rectangles, DPSS would provide a universal basis expansion. The channel model we use is described by a four-dimensional tensor of MDPSS representing channel response [14]. By modulation of the bandwidth of a set of DPSS, we achieve different scattering scenarios with parameters defined by theoretical models or/and measurements [14].

\section{是 Springer}

(c) 2014 Uchiteleva and Primak; licensee Springer. This is an Open Access article distributed under the terms of the Creative Commons Attribution License (http://creativecommons.org/licenses/by/2.0), which permits unrestricted use, distribution, and reproduction in any medium, provided the original work is properly credited. 


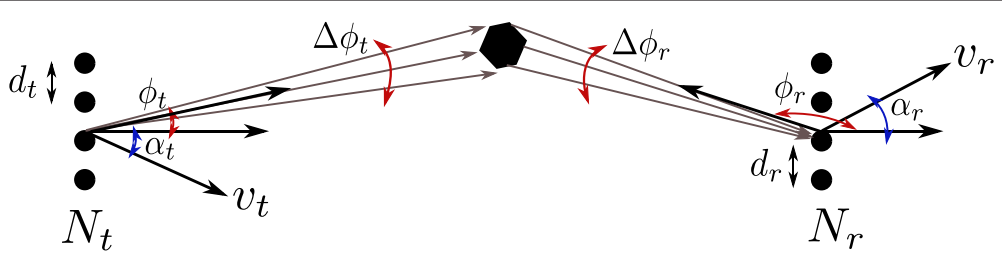

Figure 1 Geometry of a single cluster problem.

$\mathrm{V} 2 \mathrm{~V}$ communication is accompanied by the movement of both receive and transmit sides with low elevation antennas and scatterers, which are assumed to be located on perimeters of multiple co-focal ellipses (with the receiver and the transmitter at ellipses' foci). MDPSS channel model is a regular-shaped geometrybased stochastic model (RS-GBSM), which is very flexible in definition of the geometry and location of different clusters in Moderate Spatial Scale (MSS) or Small Spatial Scale (SSS) scenarios [15]. Furthermore, this model is suitable for application in both V2I and V2V scenarios, as it gives us the control over definition of the motion of both communication sides. Thereafter, we evaluate how scattering from narrow clusters affects estimation quality of the mobile. These results could be further used in analysis and optimization of IP-level protocols, such as PMIPv6 [16].

Multiple-input single-output (MISO) is a very common scenario in the downlink of a cellular system. Therefore, in our work, we focused on a simple yet elegant coding technique, the Alamouti scheme [4], which is used in some third/fourth generation wireless mobile standards. Pilot-assisted channel estimation is used with Wiener filter as a pilot filter [3]. In IEEE 802.11p, V2x communication standard single-input single-output (SISO) systems are postulated, but multiple-input multiple-output (MIMO) systems and their variations could be employed to improve the reliability of communications.

The remainder of our paper is organized as follows: in Section 2, the MDPSS-based channel model is reviewed and simulation results for one and two cluster case are presented. The $2 \times 1$ MISO communication system with Alamouti coding and channel estimation is described in Section 3. In Section 4, two different cases of environment were tested and the performance of the system was evaluated via MMSE and BER. Moreover, an example of simulation of the channel at a real intersection was shown, followed by analysis of communication link. The conclusion is in Section 5.

\section{Channel model}

\subsection{Geometry and channel response}

In order to simulate a single cluster environment, we use a geometry shown in Figure 1: there are two horizontal multi-element linear antenna arrays on both receiving and transmitting sides; the space between antennas contains a single scattering cluster. Impulse response $H(\tau, t)$ is assumed to be sampled at the rate $F_{s t}\left(\tau=n / F_{s t}\right)$ and the channel is sounded at the rate $F_{s}\left(t=m / F_{s}\right)$. The carrier frequency is $f_{c} ; N_{r}, N_{t}, d_{r}, d_{t}$ are the number of isotropic elements and the distance between them at receiving and transmitting antennas respectively; $v_{r}$ and $v_{t}$ are velocities at which receiver and transmitter move making angles $\alpha_{t}$

Table 1 Example of simulation parameters for the channel with one scattering cluster

\begin{tabular}{|c|c|c|}
\hline Param. & Value & Description \\
\hline$N_{r}$ & 8 & Number of antennas on the receiving side \\
\hline$N_{t}$ & 8 & Number of antennas on the transmitting side \\
\hline$v_{r}$ & $30 \mathrm{~km} / \mathrm{h}$ & Speed of the receiver \\
\hline$v_{t}$ & $0 \mathrm{~km} / \mathrm{h}$ & Speed of the transmitter \\
\hline W & $6 \mathrm{MHz}$ & Required channel half-bandwidth \\
\hline$f_{c}$ & $2 \mathrm{GHz}$ & Carrier frequency \\
\hline$d_{r}, d_{t}$ & 0.5 & $\begin{array}{c}\text { Receive/transmit antenna spacing normalized to } \\
\text { wave length }\end{array}$ \\
\hline$P_{c}$ & 1 & Power weights for clusters \\
\hline$\phi_{0 r}$ & $20^{\circ}$ & $\begin{array}{l}\text { Azimuthal angle at which center of the cluster } \\
\text { is seen to the receiver }\end{array}$ \\
\hline$\phi_{0 t}$ & $10^{\circ}$ & $\begin{array}{l}\text { Azimuthal angle at which center of the } \\
\text { cluster is seen to the transmitter }\end{array}$ \\
\hline$\alpha_{r}, \alpha_{t}$ & $0^{\circ}$ & $\begin{array}{l}\text { The angle between broadside vector and } \\
\text { movement direction }\end{array}$ \\
\hline$\Delta \phi_{r}$ & $5^{\circ}$ & Angular spread seen from receiving side \\
\hline$\Delta \phi_{t}$ & $8^{\circ}$ & Angular spread seen from transmitting side \\
\hline$\tau$ & $0.3 \mu \mathrm{s}$ & A mean delay associated with the cluster \\
\hline$\Delta \tau$ & $0.1 \mu \mathrm{s}$ & Corresponding delay spread \\
\hline$F_{s t}$ & $50 \mathrm{MHz}$ & Sampling frequency in delay domain \\
\hline$F_{S}$ & $250 \mathrm{~Hz}$ & Rate of sampling in Doppler domain \\
\hline irL & 142 & Length of the impulse response (num of samples) \\
\hline L & 1,024 & Number of samples (in Doppler domain) \\
\hline $\mathrm{Nf}$ & 128 & $\begin{array}{l}\text { Number of equally spaced samples for process } \\
\text { representation at bandwidth }[-W, W]\end{array}$ \\
\hline rate & $10^{5} \mathrm{bps}$ & The transmission rate, bits per second \\
\hline
\end{tabular}


and $\alpha_{r}$ with corresponding broadside vectors; $\phi_{r}$ and $\phi_{t}$ are azimuthal angles at which a cluster center is seen from receiving and transmitting sides, respectively. For simplicity, it is assumed that co-elevation angles $\theta_{t}=\theta_{r}=\frac{\pi}{2}$ and there is no spread at this direction [14]. The cluster corresponds to time delay $\tau$ with delay spread $\Delta \tau$, as well as angle of departure (AoD) and angle of arrival (AoA) $\phi_{t}, \phi_{r}$ respectively with angular spreads $\Delta \phi_{r}, \Delta \phi_{t}$ at each communication side. The Doppler shift is calculated as follows:

$$
f_{D}=\frac{f_{c}}{c}\left[v_{t} \cos \left(\phi_{t 0}-\alpha_{t}\right)+v_{r} \cos \left(\phi_{r 0}-\alpha_{r}\right)\right],
$$

and the resulting Doppler spectrum widening is

$\Delta f_{D}=\frac{f_{c}}{c}\left[v_{t} \Delta \phi_{t}\left|\sin \left(\phi_{t 0}-\alpha_{t}\right)\right|+v_{r} \Delta \phi_{r}\left|\sin \left(\phi_{r 0}-\alpha_{r}\right)\right|\right]$,

A sample of the complete MIMO channel response takes a form of four-dimensional tensor [14]:

$$
\begin{aligned}
\mathcal{H}_{4}= & \mathcal{W}_{4} \odot \sum_{n_{r}}^{D_{r}} \sum_{n_{t}}^{D_{t}} \sum_{n_{f}}^{D_{f}} \sum_{n_{d}}^{D} \sqrt{\lambda_{n_{r}}^{(r)} \lambda_{n_{t}}^{(t)} \lambda_{n_{f}}^{(\omega)} \lambda_{n_{d}}^{(d)}} . \\
& { }_{1} u_{n_{r}}^{(r)} \times_{2} u_{n_{t}}^{(t)} \times_{3} u_{n_{f}}^{(\omega)} \times_{4} u_{n_{d}}^{(d)} \cdot \xi_{n_{r}, n_{t}, n_{f}, n_{d}},
\end{aligned}
$$

where $u_{n_{r}}^{(r)}, u_{n_{t}}^{(t)}, u_{n_{f}}^{(\omega)}, u_{n_{d}}^{(d)}$ are DPSS representing dimensions of a signal at the Receive, Transmit, Frequency and Doppler domains with 'domain-dual domain' products: $\left|\Delta \phi_{r} \frac{N_{r} d_{r}}{\lambda} \cos \phi_{r 0}\right|,\left|\Delta \phi_{t} \frac{N_{t} d_{t}}{\lambda} \cos \phi_{t 0}\right|, W \Delta \tau, T_{\max } \frac{\Delta f_{D}}{2}$ respectively and $D_{r, t}=\left\lceil 2 \Delta \phi_{r, t} \frac{d_{r, t}}{\lambda} \cos \phi_{0 r, t}\right\rceil+1, D_{f}=$ $\lfloor 2 W \Delta \tau\rfloor+1, D=\left\lfloor\Delta f_{D} T_{\max }\right\rfloor+1$ the numbers of DPSS needed in each domain. $\xi_{n_{r}, n_{t}, n_{f}, n_{d}}$ are Complex Gaussian i.i.d. variables with unit variance. $\mathcal{W}_{4}$ is a tensor of modulating sinusoids described as follows:

$$
\begin{aligned}
\mathcal{W}_{4} & =1 w^{(r)} \times 2 w^{(t)} \times{ }_{3} w^{(\omega)} \times{ }_{4} w^{(d)}, \\
w^{(r)} & =\left[1, e^{\left(j 2 \pi \frac{d_{r}}{\lambda} \sin \phi_{r 0}\right)}, \ldots, e^{\left(j 2 \pi \frac{d_{r}}{\lambda} \sin \phi_{r 0}\left(N_{r}-1\right)\right)}\right]^{T}, \\
w^{(t)} & =\left[1, e^{\left(j 2 \pi \frac{d_{t}}{\lambda} \sin \phi_{r 0}\right)}, \ldots, e^{\left(j 2 \pi \frac{d_{t}}{\lambda} \sin \phi_{t 0}\left(N_{t}-1\right)\right)}\right]^{T} \\
w^{(\omega)} & =\left[1, e^{(j 2 \pi \Delta F \tau)}, \ldots, e^{\left(j 2 \pi \Delta F \tau\left(N_{f}-1\right)\right)}\right]^{T}, \\
w^{(d)} & =\left[1, e^{\left(j 2 \pi f_{D} T_{s}\right)}, \ldots, e^{\left(j 2 \pi f_{D}\left(T_{\max }-T_{s}\right)\right)}\right]^{T}
\end{aligned}
$$

where $\odot$ is element-wise (Hadamard) product of two tensors and $T_{\max }$ is the duration of the simulation. In

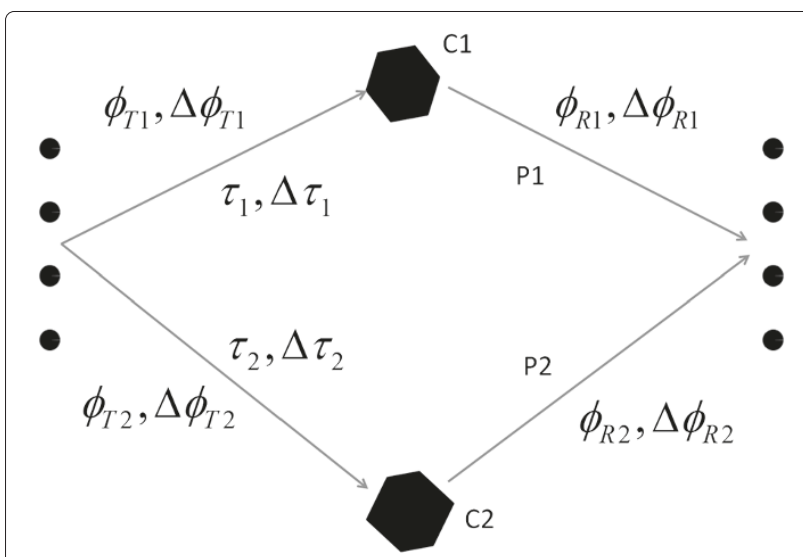

Figure 2 Two-cluster environment example.

simulation of environment with $N_{c}$ clusters the total channel response is a superposition of independently generated normalized single-cluster responses $\mathcal{H}_{4}(k)$ :

$$
\mathcal{H}_{4}=\sum_{k=1}^{N_{c}} \sqrt{P_{k}} \mathcal{H}_{4}(k), \quad P=\sum_{k=1}^{N_{c}} P_{k},
$$

where $P_{k}$ is the relative power of $k$-th cluster and $P$ is a total power. The auto-covariance function $R_{\text {tot }}(\tau)$ in this case is a sum of $N_{c}$ auto-covariance functions of individual one-cluster problems (due to linearity of the Fourier transform operation):

$$
R_{\text {tot }}(\tau)=\sum_{k=1}^{N_{c}} P_{k} \exp \left(j 2 \pi f_{D_{k}} \tau\right) \operatorname{sinc}\left(\Delta f_{D_{k}} \tau\right) .
$$

\subsection{Simulation examples}

In this section, we present some results from simulation of a flat fading channel with one and two clusters and compare them to theoretical derivations, discussed previously. An example of parameter summary of a single cluster from Figure 1 is given in Table 1, and parameters of a two-cluster case, which is depicted in Figure 2, are given in Table 2. If some parameters are not mentioned

Table 2 Two-cluster environment parameters

\begin{tabular}{lcc}
\hline Cluster & Parameter & Value \\
\hline \multirow{3}{*}{ Cluster1 } & $\phi_{0 r_{1}}$ & $10^{\circ}$ \\
& $\tau_{1}$ & $0.3 \mu \mathrm{s}$ \\
& $P_{1}$ & 0.6 \\
Cluster2 & $\phi_{0 r_{2}}$ & $120^{\circ}$ \\
& $\tau_{2}$ & $0.8 \mu \mathrm{s}$ \\
& $P_{2}$ & 0.4 \\
\hline
\end{tabular}




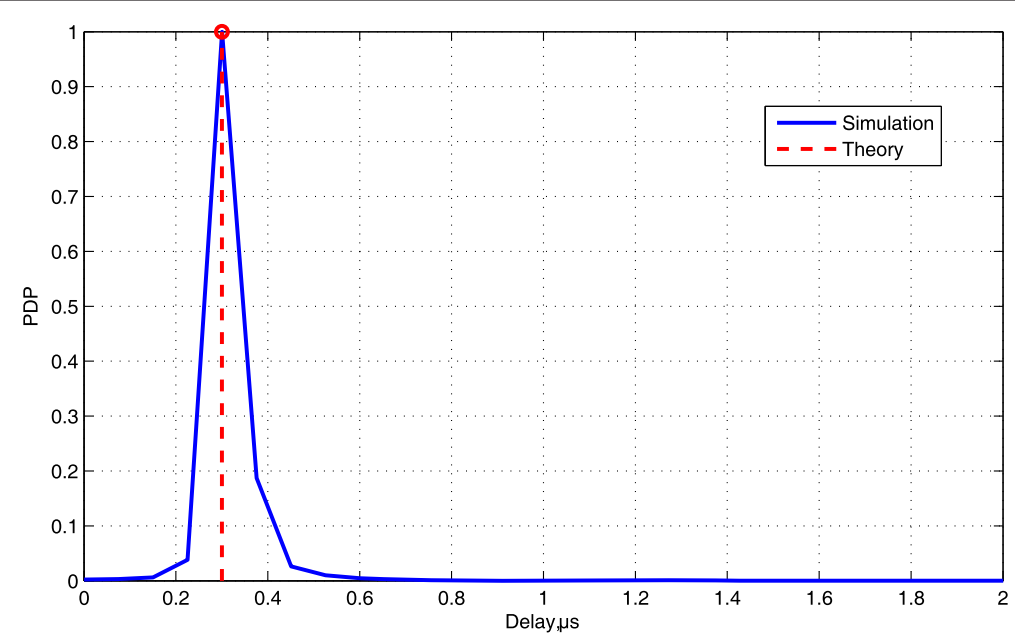

Figure 3 PDP of one-cluster channel response, $\tau=0.3 \mu \mathrm{s}$.

in the second table, they remain the same as in Table 1 and equal for both clusters. Simulation results for the onecluster scenario are given in Figures 3, 4, and 5. Power delay profile (PDP) of this case is given in Figure 3, where there is a clear peak at delay associated with a particular cluster $\tau=0.3 \mu$ s with delay spread of $\Delta \tau=0.1 \mu \mathrm{s}$. At Figure 4, we may see power spectral density (PSD) with resulting widened Doppler spectrum around frequency $f_{D} \approx 54.7 \mathrm{~Hz}$ with Doppler spread of $\Delta f_{D}=4.8 \mathrm{~Hz}$ (calculated from Equations 1 and 2). The absolute value of auto-covariance function for this case, $|R(\tau)|$, is given in Figure 5 as a function of normalized Doppler time $f_{D_{0}} \tau$, where $f_{D_{0}}=f_{c} \frac{\left|v_{t}\right|+\left|v_{r}\right|}{c}$. In all figures, we can see a good correspondence between the theory and the simulation.
PDP and PSD of a two-cluster environment are shown in Figures 6 and 7, where we may observe a pick of received power at delays $\tau_{1}=0.3 \mu$ s and $\tau_{2}=0.8 \mu$ s with power delay spread of $0.1 \mu \mathrm{s}$ each (Figure 6) and widening of spectrum at $f_{D 1} \approx 55 \mathrm{~Hz}$ and $f_{D 2} \approx-28 \mathrm{~Hz}$ (Figure 7), the same frequencies that one may calculate from Equation 1. Theoretical and simulated envelopes of auto-covariance function are plotted in Figure 8. Again, there is a good agreement between simulation and theory.

\section{Transmission system}

We simulate the $2 \times 1$ MISO system with binary phase shift keying (BPSK) modulation and Alamouti coding scheme. Two transmitting antennas are assumed to be far enough from each other (the distance should be at least

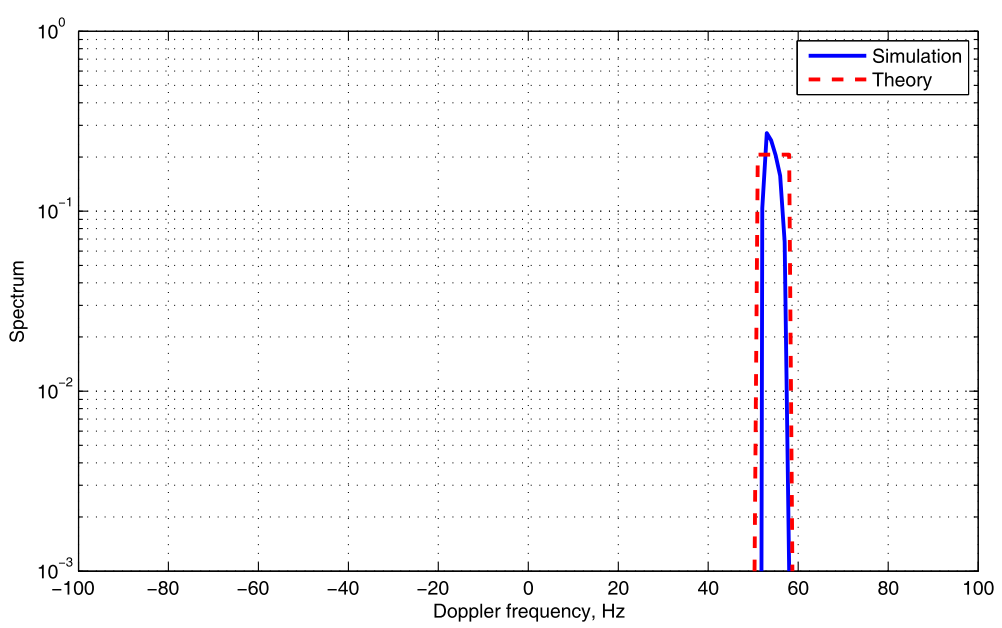

Figure 4 Doppler PSD of one-cluster channel response, $f_{D} \approx 54.7 \mathrm{~Hz}$. 


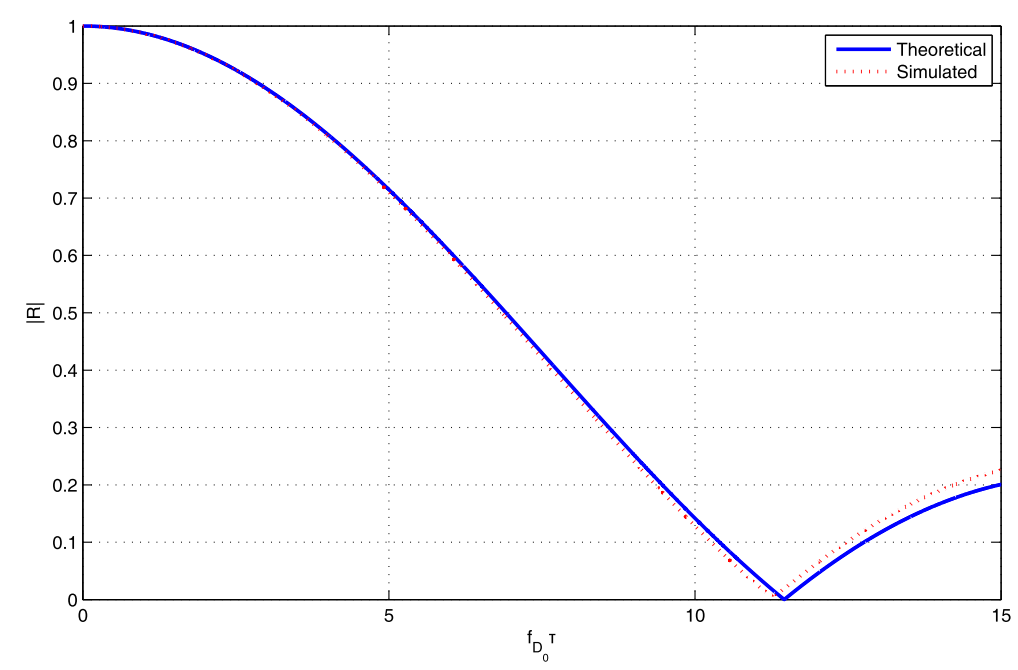

Figure 5 Envelope of the auto-covariance function of the channel process, $|R(\tau)|$.

10 times greater than the carrier wavelength), so that each resolvable pass fades independently. For each symbol time the baseband equivalent model at the receiver is [4]:

$$
y[m]=h_{1}[m] x_{1}[m]+h_{2}[m] x_{2}[m]+\xi[m],
$$

where $h_{j}[m]$ is a flat-fading Rayleigh channel gain between the transmitting antenna $j(j=1,2)$ and the receiver, and $\xi[m]$ is the sampled additive white Gaussian noise, $\xi[m] \sim \mathcal{C N}\left(0, N_{0}\right)$. In the Alamouti encoder [4], data stream is separated into two symbol blocks and sent from two antennas with rate of two bits per two symbol times. Two complex symbols $u_{1}$ and $u_{2}$ are transmitted in the following order:
(1) At first symbol time, $x_{1}[1]=u_{1}, x_{2}[1]=u_{2}$ are transmitted

(2) At second symbol time, $x_{1}[2]=-u_{2}^{*}, x_{2}[2]=u_{1}^{*}$ are transmitted

(3) It is also assumed that the channel remains constant over two symbol times: $h_{1}[1]=h_{1}[2]=h_{1}$, $h_{2}[1]=h_{2}[2]=h_{2}$ (the quasi-static channel assumption is valid when data rates are relatively high).

See Figure 9 for the visualization. Equation 8 could be rewritten in a matrix form [4]:

$$
[y[1] y[2]]=\left[\begin{array}{ll}
h_{1} & h_{2}
\end{array}\right]\left[\begin{array}{rr}
u_{1} & -u_{2}^{*} \\
u_{2} & u_{1}^{*}
\end{array}\right]+[\xi[1] \xi[2]]
$$

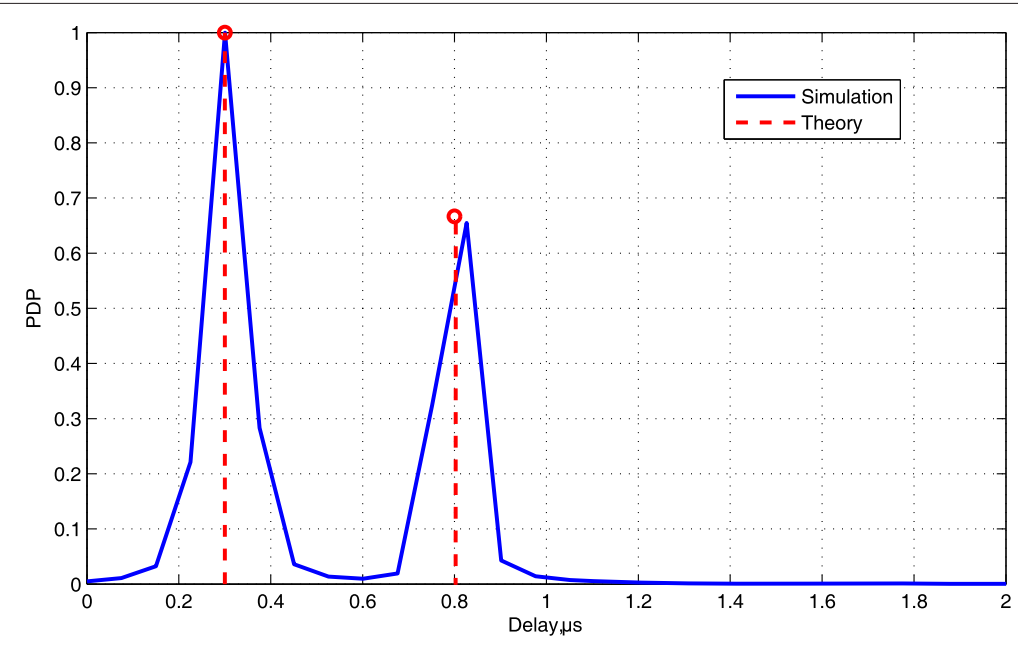

Figure 6 PDP of a two-cluster case, $\tau_{1}=0.3 \mu \mathrm{s}, \tau_{2}=0.8 \mu \mathrm{s}$. 


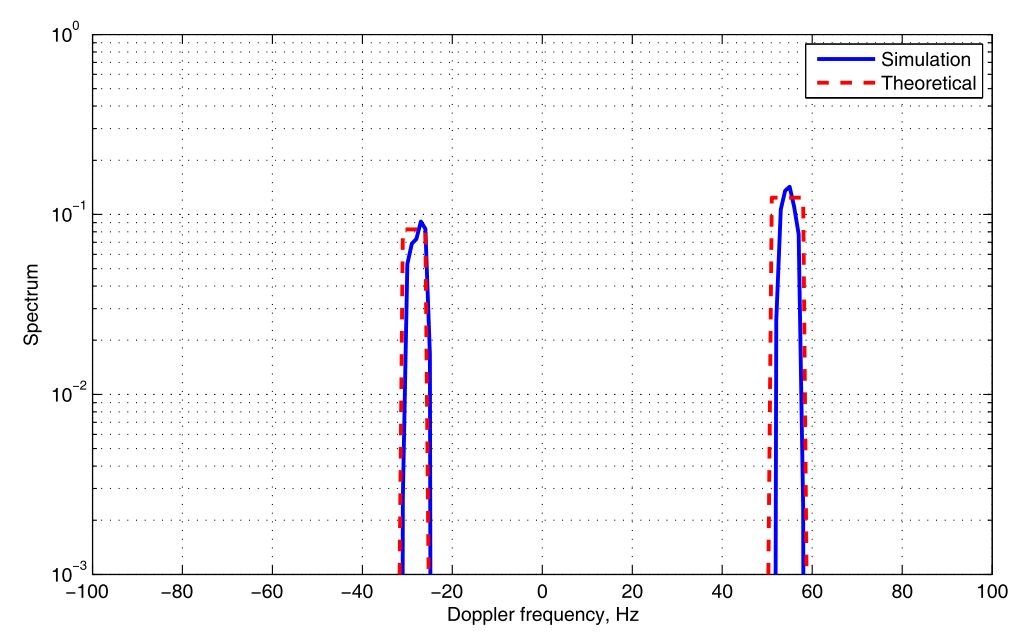

Figure 7 Doppler PSD of two-cluster channel, $f_{D_{1}} \approx 55.7 \mathrm{~Hz}, f_{D_{2}} \approx-27.8 \mathrm{~Hz}$.

or after some rearrangement,

$$
\left[\begin{array}{l}
y[1] \\
y[2]^{*}
\end{array}\right]=\left[\begin{array}{rr}
h_{1} & h_{2} \\
h_{2}^{*} & -h_{1}^{*}
\end{array}\right]\left[\begin{array}{l}
u_{1} \\
u_{2}
\end{array}\right]+\left[\begin{array}{l}
\xi[1] \\
\xi[2]^{*}
\end{array}\right]=\mathbf{H u}+\boldsymbol{\xi} .
$$

The columns of the square matrix $\mathbf{H}$ are orthogonal, hence one may separate Equation 10 into two orthogonal problems. To decode information $\mathbf{y}$ is projected onto each of the two columns of the matrix: $\left[h_{1} h_{2}^{*}\right]^{t},\left[\begin{array}{ll}h_{2} & -h_{1}^{*}\end{array}\right]^{t}$ :

$$
r_{j}=\|\mathbf{h}\| u_{j}+\xi_{j}, \quad i=1,2,
$$

where $\mathbf{h}=\left[h_{1}, h_{2}\right]^{t}$ and $\xi_{j} \sim C N\left(0, N_{0}\right)\left(\xi_{1}, \xi_{2}\right.$ are independent), and then ML detection is performed for each of the decoded signals. The exact bit error probability for BPSK modulation was derived in [3] for a limiting case of perfect CSI and fully correlated channel gains, i.e. $R(\tau)=$ 1 , at the receiver:

$$
P_{b}=\frac{1}{4}\left(2+\sqrt{\frac{\bar{\gamma}_{\mathrm{s}}}{\bar{\gamma}_{\mathrm{s}}+2}}\right)\left(1-\sqrt{\frac{\bar{\gamma}_{\mathrm{s}}}{\bar{\gamma}_{\mathrm{s}}+2}}\right)^{2}
$$

$\bar{\gamma}_{s}$ is the average data signal-to-noise ratio (SNR) or $E_{b} / N_{0}$, when $\sqrt{E_{b}}$ is the amplitude of the signal.

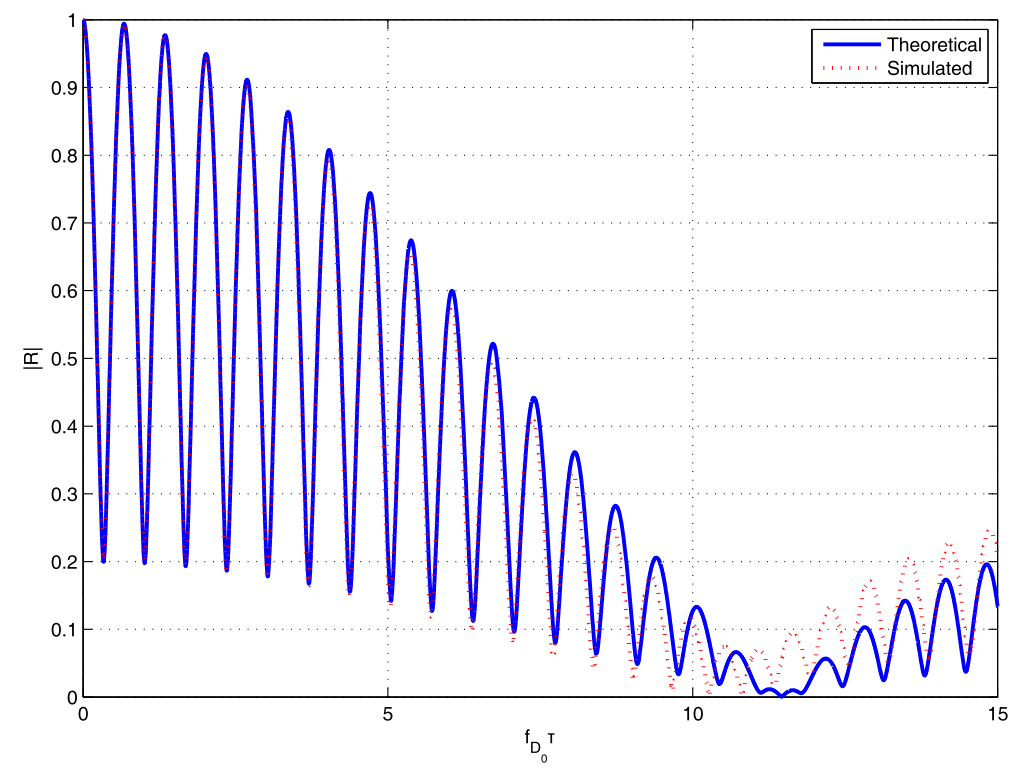

Figure 8 Envelope of auto-covariance function of two-cluster channel process, $|R(\tau)|$. 

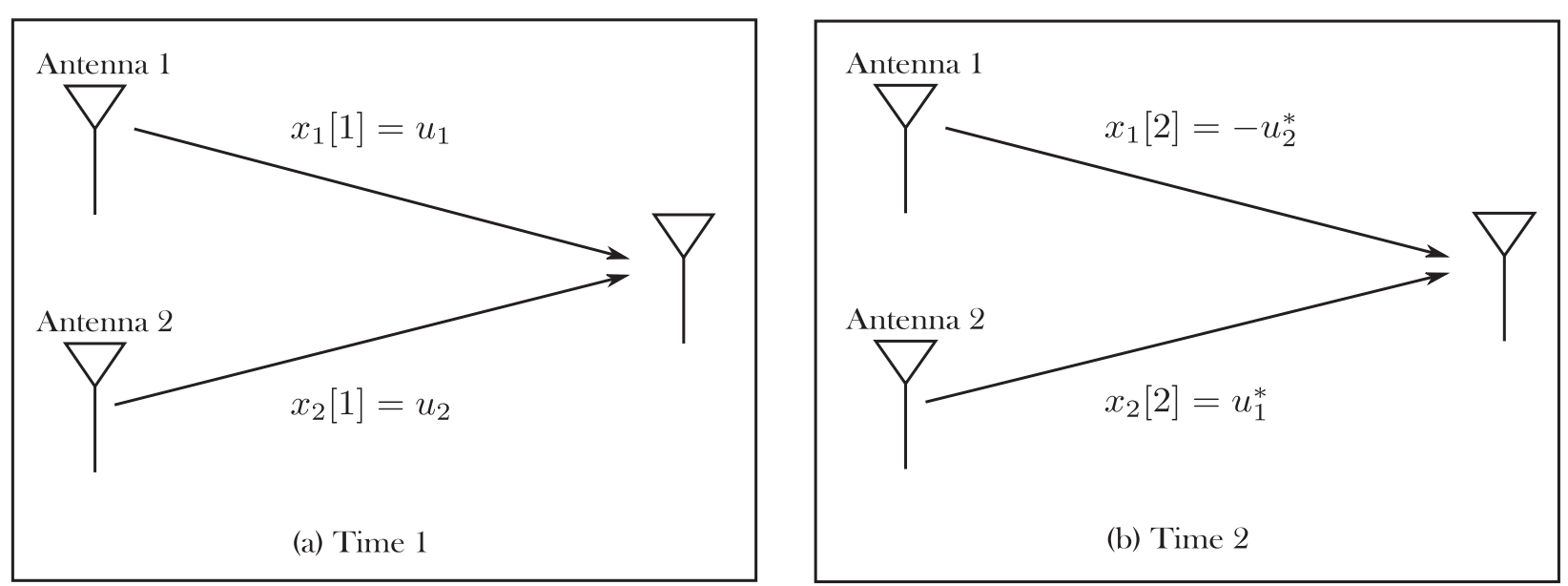

Figure 9 Alamouti coding at time 1 (a) and time 2 (b).

As the perfect CSI is not available in real-life communication, estimation of channel gains at the receiver is always required. We use pilot-assisted scheme with Wiener filter at the receiver $[1,3,17]$. The information codewords (or blocks of two symbol time lengths each) are divided into frames and interleaved with pilot symbols. Each frame contains $N_{b}+1$ blocks: $N_{b}$ blocks of information symbols and one block of pilot which is added at the beginning of the frame. The receiver possesses the information about bit rate and the frame length. Therefore, it is able to extract pilot signals from the data stream and store them in the buffer of length $2 M+1$, where $M$ is an integer, which represents the number of pilots in the 'future' and 'past', i.e. the estimation and decoding processing is performed with delay of $M$ frame times. An example of a division into frames and pilot interleaving is visualized in Figure 10. Based on the information from the buffer and known channel statistics, the receiver performs channel estimation, decoding and decision. In addition to the baseband representation (8), we express the pilot signal $r_{p}$ (which has energy 1 , or $1 / 2$ per antenna) and the buffer $\overrightarrow{\mathbf{r}}_{p}$ for each antenna as follows [3]:

$$
\begin{aligned}
& r_{p}^{j}[m]=\frac{1}{\sqrt{2}} h_{j}[m]+\xi_{j}[m] \\
& \overrightarrow{\mathbf{r}}_{p}^{j}[m]=\left[r_{p}^{j}[m-M] \cdots r_{p}^{j}[m] \cdots r_{p}^{j}[m+M]\right]^{T}
\end{aligned}
$$

Due to channel variations in time, the filter coefficients should be recalculated every symbol slot within the frame, therefore they take the following form [3]:

$$
\mathbf{h}_{e}=\frac{1}{\sqrt{2}}\left(\frac{\mathbf{D}_{0}}{2}+\bar{\gamma}_{p}^{-1} \mathbf{I}_{2 M+1}\right)^{-1} \boldsymbol{\rho}_{e}
$$

$\mathbf{D}_{e}$ denotes a square matrix of size $(2 M+1)$ with entries given by:

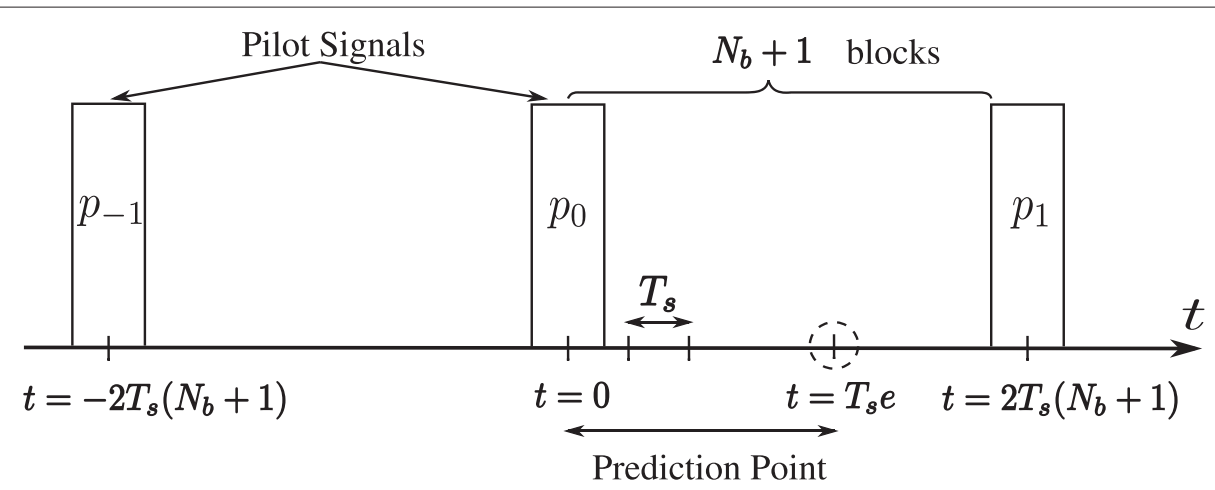

Figure 10 An example of a frame with 3 pilot signals, $M=1$. 


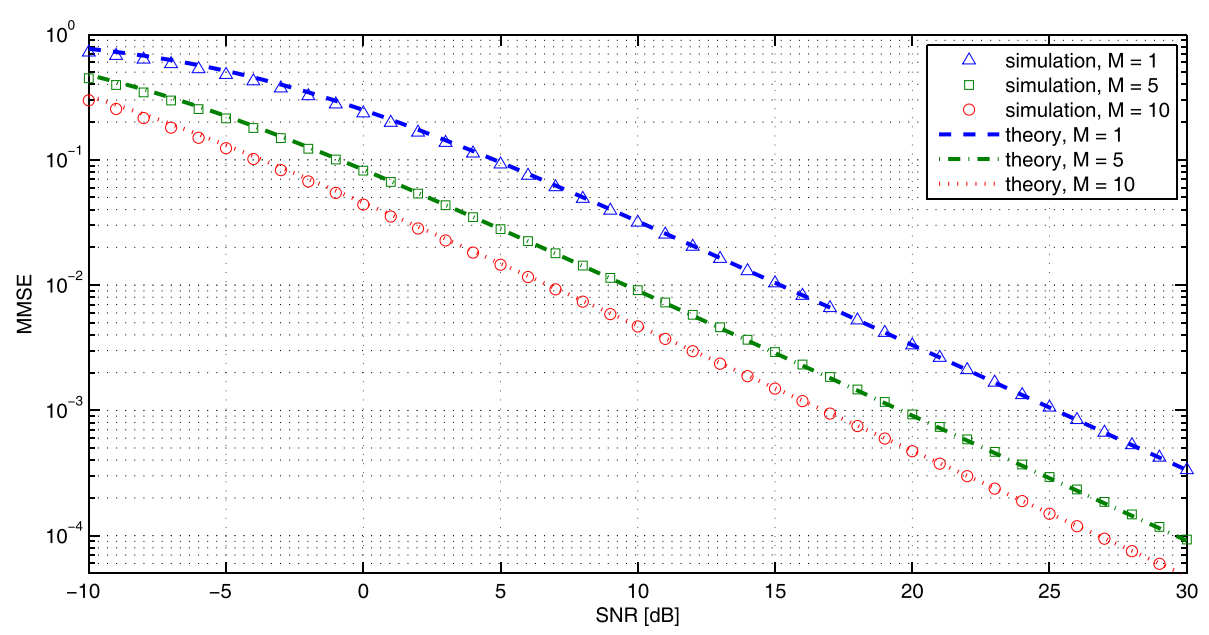

Figure 11 Estimation quality as a function of SNR and number of pilot signals $M$.

$$
\begin{aligned}
\mathbf{D}_{e}(k, l) & =R\left(-e T_{s}+(k-l) T_{s} N_{b}\right), \\
k, l & =1, \ldots, 2 M+1 ; e=0, \ldots, 2 N_{b}+1 .
\end{aligned}
$$

$\mathbf{I}_{2 M+1}$ is identity matrix of size $(2 M+1), \bar{\gamma}_{p}$ denotes the average pilot SNR or pilot $E_{p} / N_{0}, R(\cdot)$ is channel autocovariance function and $\boldsymbol{\rho}_{e}$ is the $(M+1)^{\text {th }}$ column of $\mathbf{D}_{e}$. Thus, the matrix $\mathbf{D}_{0}$ is a correlation matrix between pilot signals given in the buffer, and $\rho_{e}$ is a vector of correlations between the frame element at place $e$ and the nearest pilot signals. In a case of a static channel $\mathbf{D}_{e}=\mathbf{D}_{0}$ and $\rho_{e}=\rho_{0}$ for any $e$. Estimated channel gains for antenna 1 and 2 are given by:

$$
\hat{h}_{1}[m, e]=\mathbf{h}_{e}^{H} \mathbf{r}_{p}^{1}[m], \quad \hat{h}_{2}[m, e]=\mathbf{h}_{e}^{H} \mathbf{r}_{p}^{2}[m] .
$$

Index $m$ runs on frame slots with interval $2\left(N_{b}+1\right) T_{s}$. It is worth mentioning that since we deal with Gaussian distributed channel gains, Wiener filter is an optimal estimation filter.

BER of this scheme has been derived in [3] and is a function of SNR and correlation between the pilots at $e=0$ as the best-case scenario:

$$
P_{e=0}=\frac{1}{4}(2+\Upsilon)(1-\Upsilon)^{2}
$$

Here

$$
\Upsilon=\left(\frac{4\left(1+\bar{\gamma}_{s}^{-1}\right)}{\epsilon_{0}}-\left(\frac{\epsilon_{1}}{\epsilon_{0}}\right)^{2}\right)^{-\frac{1}{2}}
$$

and

$$
\begin{aligned}
& \epsilon_{0}=\boldsymbol{\rho}_{0}^{H}\left(\frac{\mathbf{D}_{0}}{2}+\bar{\gamma}_{p}^{-1} \mathbf{I}_{2 M+1}\right)^{-1} \boldsymbol{\rho}_{0}, \\
& \epsilon_{1}=\boldsymbol{\rho}_{1}^{H}\left(\frac{\mathbf{D}_{0}}{2}+\bar{\gamma}_{p}^{-1} \mathbf{I}_{2 M+1}\right)^{-1} \boldsymbol{\rho}_{0} .
\end{aligned}
$$

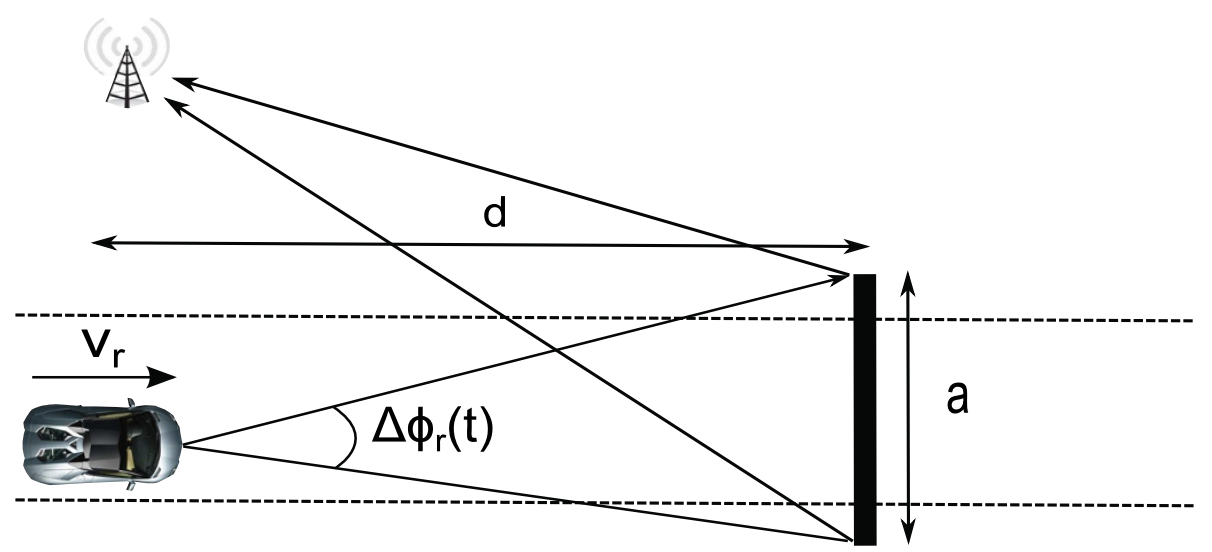

Figure 12 V2I scenario, a mobile is moving under a big road sign. 


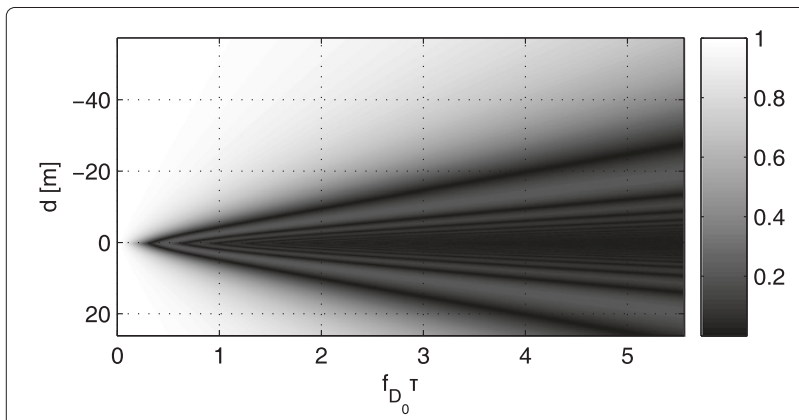

Figure 13 Absolute value of autocorrelation function of channel gains, $\left|R\left(d, f_{D_{0}} \tau\right)\right|, \mathrm{V} 2$ I scenario.

In case of perfect CSI, $R(\tau)=1$; therefore, (17) reduces to (12). Theoretical MMSE is given by

$$
\sigma_{e}^{2}=1-\boldsymbol{\rho}_{e}^{H}\left(\frac{\mathbf{D}_{0}}{2}+\bar{\gamma}_{p}^{-1} \mathbf{I}_{2 M+1}\right)^{-1} \boldsymbol{\rho}_{e}
$$

\section{Simulation results of system performance in different scenarios}

In this section, we present some numerical results which evaluate the behaviour of the transmission system, discussed in the previous section, in terms of estimation MMSE and BER, and analyze the influence of realistic scattering on the estimation quality. Thus, for example, Figure 11 shows estimation error for different number of pilots $M$ in a one-cluster environment. The simulation was performed for the rate of $50 \mathrm{Kbps}, N_{b}=5, e=0$ and cluster parameters given in Table 1 . As we would expect, the greater number of pilots reduces estimation error for any SNR. It happens because the larger number of pilots provides more information to the receiver about correlation of channel gains; therefore, better estimation is achieved. There is a good convergence between theory and simulation.

Assuming that the exact geometry description of clusters and obstacles is available through different accessible applications like Google Maps ${ }^{\odot}$ for 3D street view or through different global navigation and positioning satellite systems like GPS, GLONASS or QZSS, it is possible to model the geometry of any site of interest. Further, we present different scenarios of $\mathrm{V} 2 \mathrm{I}$ and $\mathrm{V} 2 \mathrm{~V}$ cases.

\subsection{V2I communication scenario}

V2I example is shown in Figure 12. At this scenario, we assume that a mobile, for example a car, is moving along the road and passing under a big road sign. The base station is assumed to be far away. In this case, the angular spread $\Delta \phi_{r}$ changes as a function of time (or distance to the cluster) and, as we may see from the figure $\Delta \phi_{r}$, increases as the car approaches the cluster. The expression for the varying angular spread is then:

$$
\Delta \phi_{r}(t)=2 \tan ^{-1}\left(\frac{a \tan \left(\frac{\Delta \phi_{r 0}}{2}\right)}{a-2 v_{r} t \cdot \tan \left(\frac{\Delta \phi_{r 0}}{2}\right)}\right) .
$$

Here, $\Delta \phi_{r 0}=5^{\circ}, \phi_{r} \approx 0^{\circ}$. The width of the road sign $a=5 \mathrm{~m}$ and all the other parameters are as in Table 1. Figure 13 shows behaviour of absolute value of auto-covariance function of channel gains as a function of Doppler time and a distance to the cluster. Negative distance implies that the mobile is located on the left side of

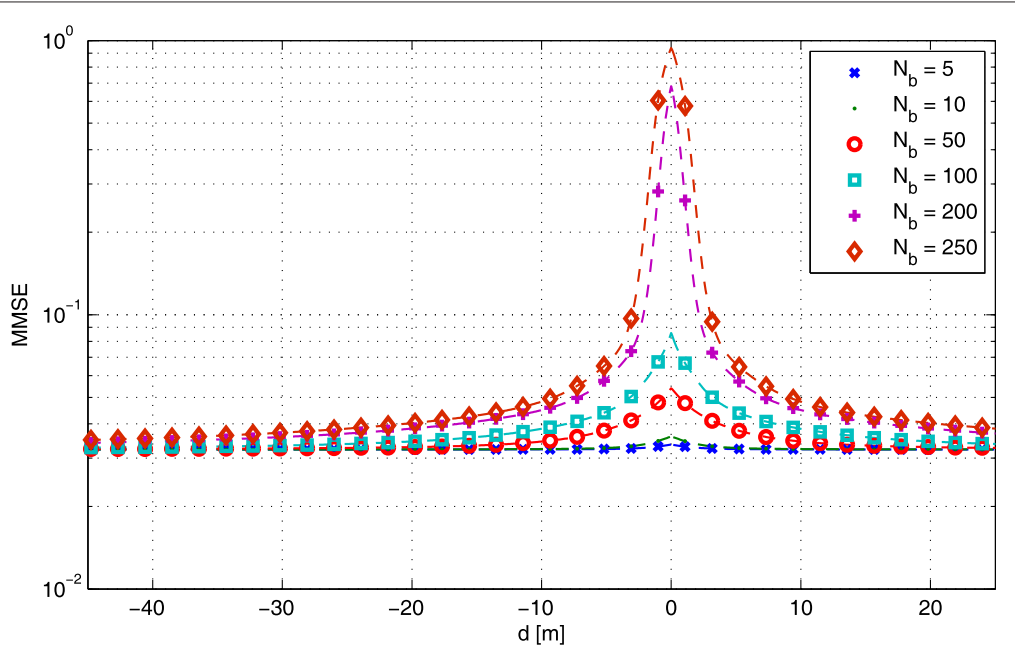

Figure 14 Estimation MMSE as a function of distance to the cluster and frame length. At SNR $=10 \mathrm{~dB}, 50 \mathrm{Kbps}$ rate and three pilot-based estimation, V2I scenario. 


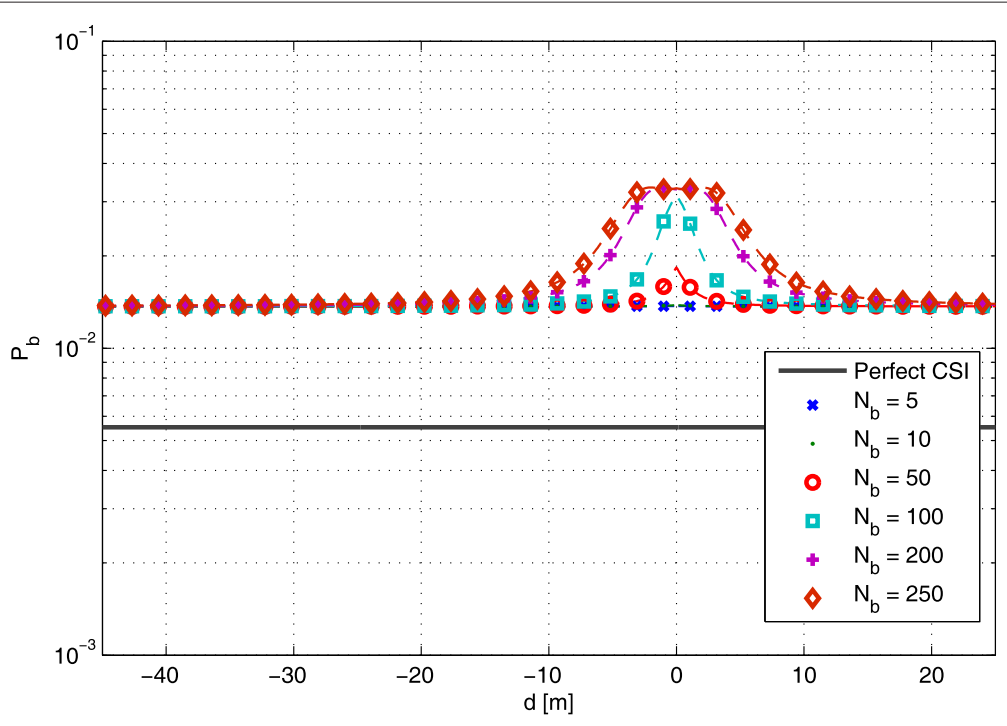

Figure $15 \mathrm{BER}$ as a function of distance to the cluster. At $\mathrm{SNR}=10 \mathrm{~dB}, 50 \mathrm{Kbps}$ rate and three pilot-based estimation, V2l scenario.

the cluster (according to Figure 12) and positive distance implies that it is located to the right. Cluster is located at $d=0 \mathrm{~m}$. It can be seen, that, as the mobile gets closer to the cluster, auto-covariance function decays faster. As a consequence, the pilot signals become less correlated, which results into higher estimation error. The behaviour of channel gains estimation MMSE as a function of distance to the cluster at $S N R=10 \mathrm{~dB}$ and $50 \mathrm{Kbps}$ rate with $M=1$ and $e=0$ for the estimation is shown in Figure 14 and the resulting BER is given in Figure 15. If we compare BER curves to Perfect CSI case (or error-free estimation), we may see the initial increase of $4 \mathrm{~dB}$ in BER, which happens because of the estimation based on three pilots only. It can be improved with use of more pilots (up to 10). Further increase in BER is introduced as the car nears the cluster. We may observe, that the effect of cluster's presence is more pronounced at longer frames. If the frame is built of more than 100 blocks, MMSE increases dramatically when the vehicle approaches the cluster. For example, for 200 block frames, the increase in MMSE is more than 10 times with resultant increase in BER by $4.7 \mathrm{~dB}$ (see Figure 15), when the car is under the road sign. Therefore, this kind of clusters produce significant shadowing effect on communication session. On the other hand, this apparent decrease of communication quality is fleeting and does not last more than a couple of seconds (in a current setting).

\subsection{V2V communications scenario}

The example of V2V scenario is shown in Figure 16. Now, the receiver and the transmitter are moving at the same direction and are passing two identical clusters, which are located on the side of the road. For simplicity,

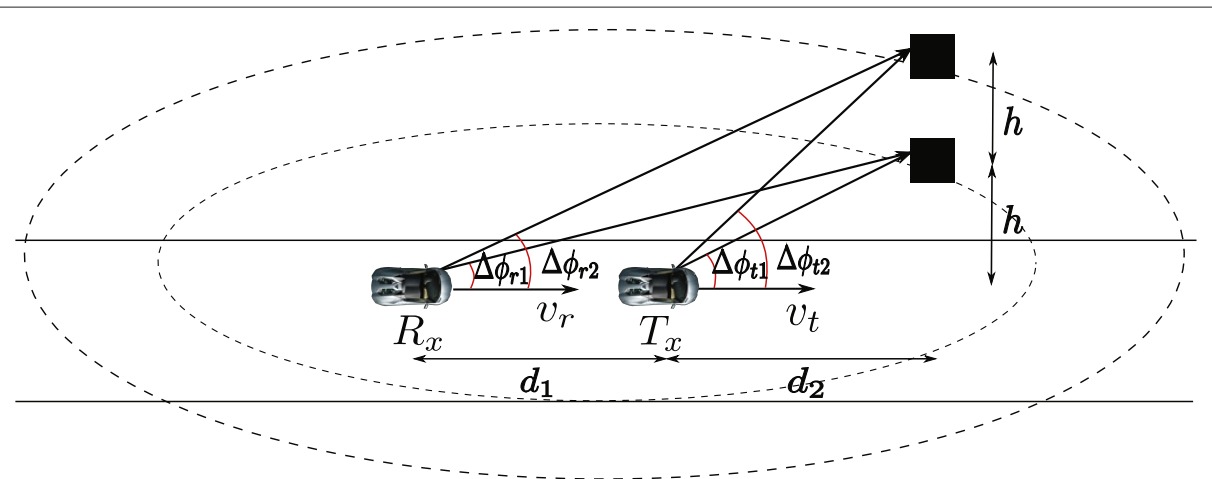

Figure 16 V2V scenario with two clusters. 


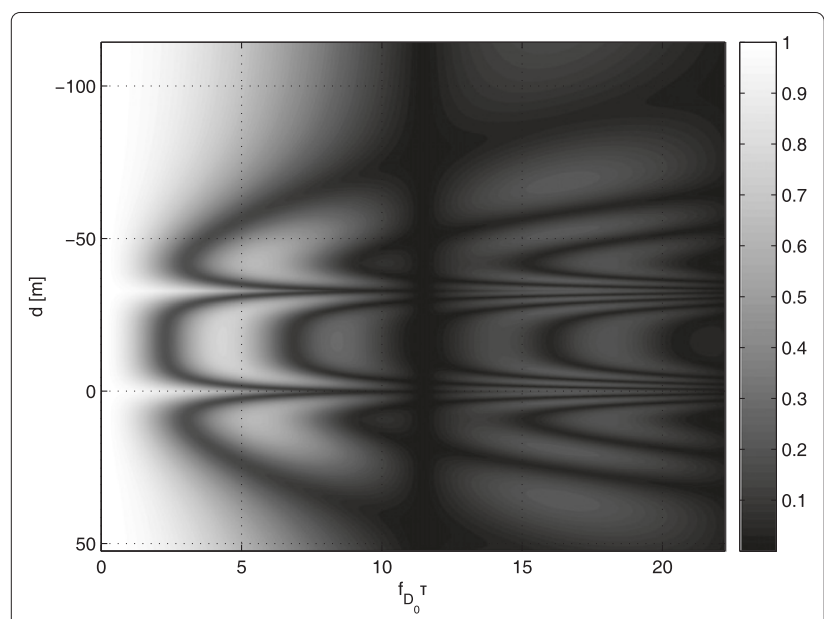

Figure 17 Absolute value of auto-covariance function of channel gains, $\left|R\left(d, f_{D_{0}} \tau\right)\right|, \mathrm{V} 2 \mathrm{~V}$ scenario.

we assume that both clusters are located on the same perpendicular to the mobile movement vector with equal distance between each other and the road, $h=10 \mathrm{~m}$. Further, we assume that the angular spread of both clusters is the same and approximately does not change as mobiles pass by, $\Delta \phi_{1}, \Delta \phi_{2} \approx 5^{\circ}$. More complicated cases without identical clusters and varying angular spread are straight forward. The initial angles between the first cluster centre and the vectors of movement of the receiver and the transmitter respectively are $\phi_{r 1_{0}}=5^{\circ}, \phi_{t 1_{0}}=7^{\circ}$. Hence, the angle between mobile movement vectors and the center of the second cluster is calculated from: $\phi_{r 2_{0}, t 2_{0}}=$ $\tan ^{-1}\left(2 \tan \left(\phi_{r 1_{0}, t 1_{0}}\right)\right)$ and equals approximately $10^{0}$ and $14^{0}$. Each one of angles $\phi_{r 1}(t), \phi_{r 2}(t), \phi_{t 1}(t), \phi_{t 2}(t)$ changes in accordance with distance change (as a function of time) between clusters and the car:

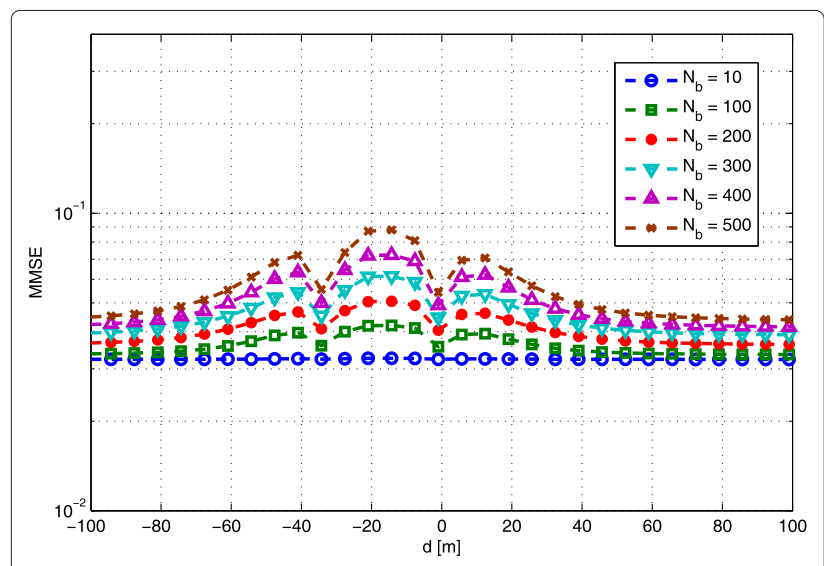

Figure 18 Estimation MMSE as a function of distance to the cluster. With respect to $R_{x}$ and a frame length $N_{b}$ at $\mathrm{SNR}=10 \mathrm{db}$ and three pilot-based estimation, V2V scenario.

$$
\phi_{r, t, i}(t)=\tan ^{-1}\left(\frac{h \tan \left(\phi_{r 0, t 0, i}\right)}{h-v t \tan \left(\phi_{r 0, t 0, i}\right)}\right), i=1,2
$$

The snapshot of auto-covariance function in the case when both vehicles' speeds equal $30 \mathrm{~km} / \mathrm{h}$ is shown in Figure 17. Resulting estimation MMSE and BER are shown in Figures 18 and 19. Figure 18 shows estimation error as a function of frame length $N_{b}$ and the distance from the cluster with respect to the receiver. As we can see, longer frames increase MMSE due to decreasing correlation between pilots. On the graph we can distinguish two notches at $d=-40 \mathrm{~m}$ and $d=0 \mathrm{~m}$, where the system experiences quick decrease in estimation MMSE, corresponding to the vehicles' location strictly perpendicular to clusters. This behaviour has a simple explanation: when one of the mobiles is located at the minimal distance to clusters, both clusters have equivalent angular parameters, what in terms of auto-covariance function equals to summation of two equally modulated sink functions; therefore, when absolute value is taken, it behaves like a one-cluster case: a slow decay in correlation as an absolute value of a pure sink function. Or effectively, the mobile 'sees' one cluster with unity power. The greatest estimation error is induced when vehicles are located at the different sides of the cluster $(-40 \mathrm{~m} \leq d \leq 0 \mathrm{~m}$ on the graph). From the graph of BER (Figure 19), we see that (as in V2I case), there is an initial recession of $4 \mathrm{~dB}$ in performance because of estimation based on three pilot signals, and further increase of $3.8 \mathrm{~dB}$ is introduced because of the cluster presence. In this scenario, the effect of clusters is not fleeting as in the previous case (V2I scenario) and starts affecting the communication quality, when the mobile is located as far as $80 \mathrm{~m}$ from the cluster.

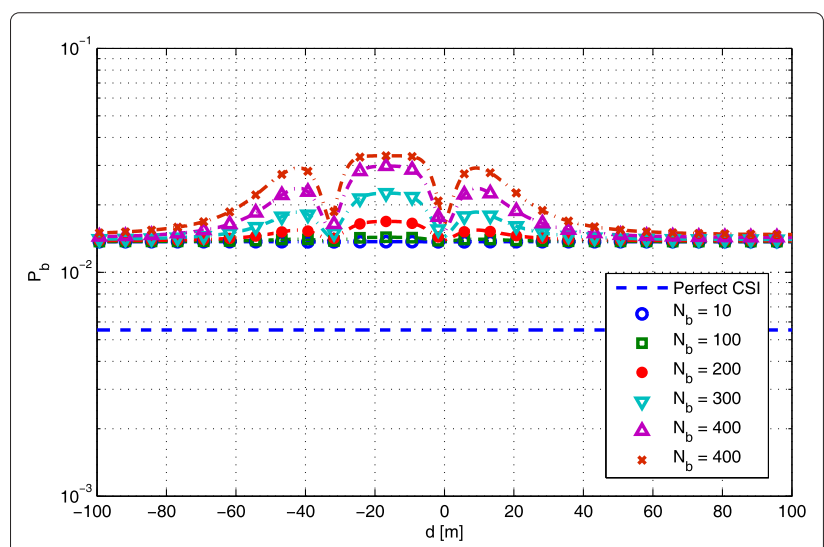

Figure $19 \mathrm{BER}$ as a function of distance to the cluster. With respect to $R_{x}$ and a frame length $N_{b}$ at the rate $S N R=10 \mathrm{db}$ and three pilot-based estimation, V2V scenario. 


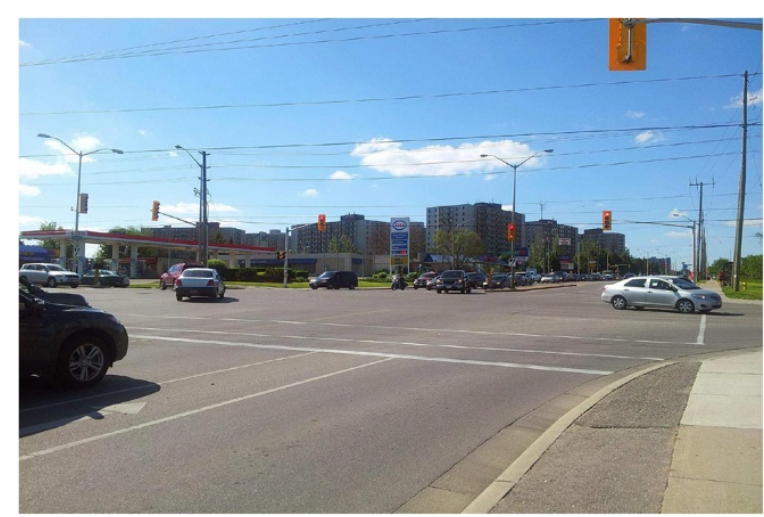

Figure 20 Real Street View, Wonderland Rd. and Oxford St. intersection, London, Ontario, Canada, ON N6H.

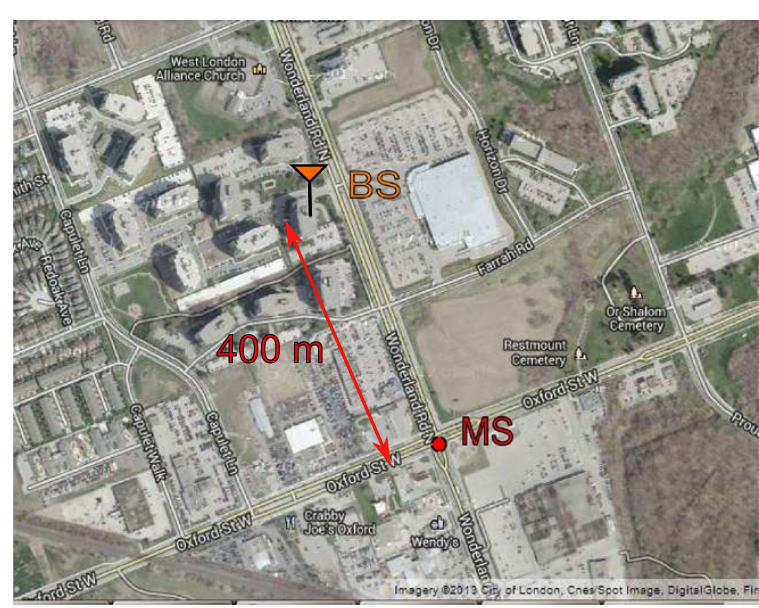

Figure 21 Google Map Street View $^{\odot}$, locations of base station and mobile station.

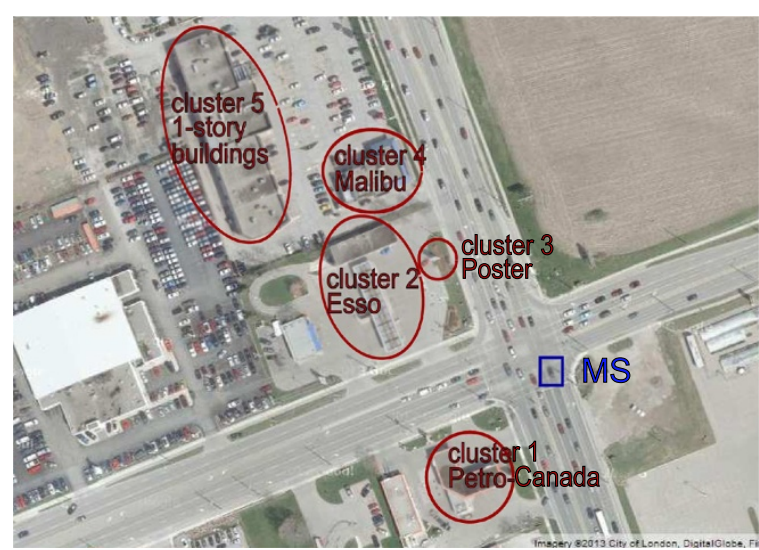

Figure 22 Google Map Street View ${ }^{\odot}$, clusters contributing to the signal scattering around the mobile. 


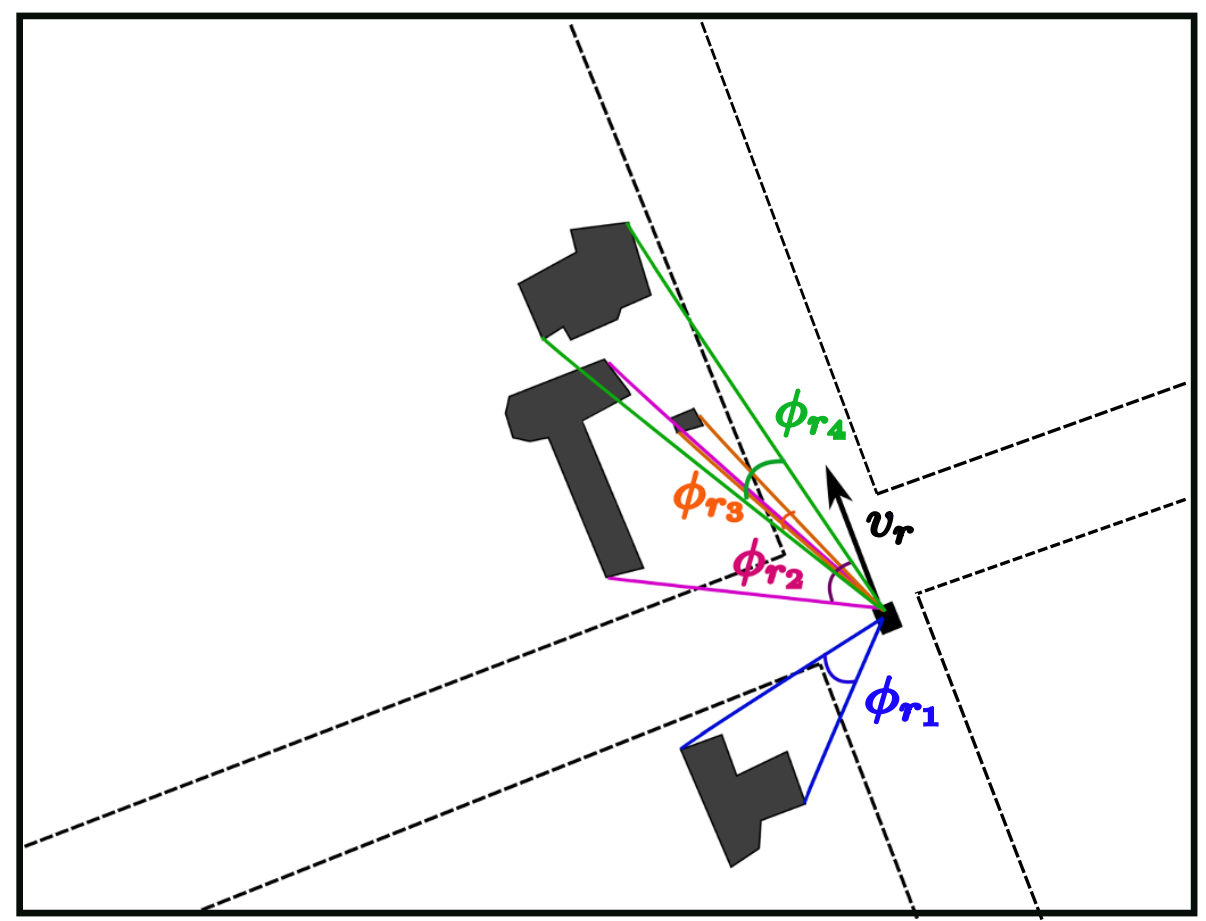

Figure 23 The geometry of the site around the mobile.

\subsection{Simulation of a real intersection in V2I case}

In this section, we show how the channel model, discussed previously, can be used for simulation of a communication link at the real-life site, located at the intersection of Wonderland Road and Oxford Street at London, Ontario, Canada, as shown in Figure 20 (East of Wonderland Road view to the North-West). In this example, we used Google Maps $^{\odot}$ application for measurements of distances and cluster dimensions due to its accessibility, but any other mapping and location application can be used for the similar analysis. Let us assume, that a mobile, equipped with the discussed communication system, is passing through the intersection with a speed of $30 \mathrm{~km} / \mathrm{h}$ and moving to the North along Wonderland Road. Let us assume as well that there is a downlink between the mobile and a cellular tower located on the roof of one of the buildings on the left-hand side of the road, at the address 720 Wonderland $\mathrm{Rd}$., which is approximately at the distance of $400 \mathrm{~m}$ to the North from the intersection, see Figure 21 (a view on the intersection at the Google $\mathrm{Maps}^{\odot}$ ). Analyzing the site, we may identify several clusters in the vicinity of the mobile: Petro-Canada and Esso gas stations on the Western side of Wonderland Road (and on opposite sides of Oxford), a big metal poster, a convenience store near the Esso gas station, and Malibu Restaurant West Inc. to the North from Esso, see Figure 22. All the rest of the buildings and obstacles are either shadowed by these four clusters (for example, cluster 5 at Figure 22) or too far to contribute to the signal scattering with respect to the current mobile location (but might be taken into consideration when recalculating the communication site layout as the mobile moves forward and approaches them). The distances, angles and angular spreads of each cluster can be easily measured and calculated using Google Distance Measurement Tool $^{\odot}$. The final cluster layout is shown in Figure 23, and parameters of each cluster are listed in Table 3 . Powers of clusters were chosen arbitrary for simplicity purposes, but could be verified through more elaborate calculations, for example with use of Radar Equation [18]. Auto-correlation function of the channel in this scenario is shown in Figure 24 as a function of normalized Doppler time and distance to

Table 3 Parameters of clusters in scenario on Wonderland Road and Oxford Street intersection

\begin{tabular}{lcccc}
\hline & Cluster $\mathbf{1}$ & Cluster 2 & Cluster 3 & Cluster 4 \\
\hline$\phi_{r}\left({ }^{\circ}\right)$ & 121.3 & 46.1 & 26.3 & 22 \\
$\Delta \phi_{r}\left(^{\circ}\right)$ & 25.7 & 35.7 & 2.4 & 16.7 \\
$\phi_{t}\left({ }^{\circ}\right)$ & 134.8 & 143.9 & 134.3 & 134.9 \\
$\Delta \phi_{t}\left(^{\circ}\right)$ & 3.4 & 5.1 & 0.6 & 6 \\
$\tau(\mu \mathrm{s})$ & 1.68 & 1.43 & 1.38 & 1.38 \\
$\Delta \tau(\mu \mathrm{s})$ & 0.09 & 0.08 & 0.003 & 0.01 \\
$h(\mathrm{~m})$ & 55.7 & 64.55 & 31.88 & 42.78 \\
$P$ & 0.3 & 0.3 & 0.1 & 0.3 \\
\hline
\end{tabular}




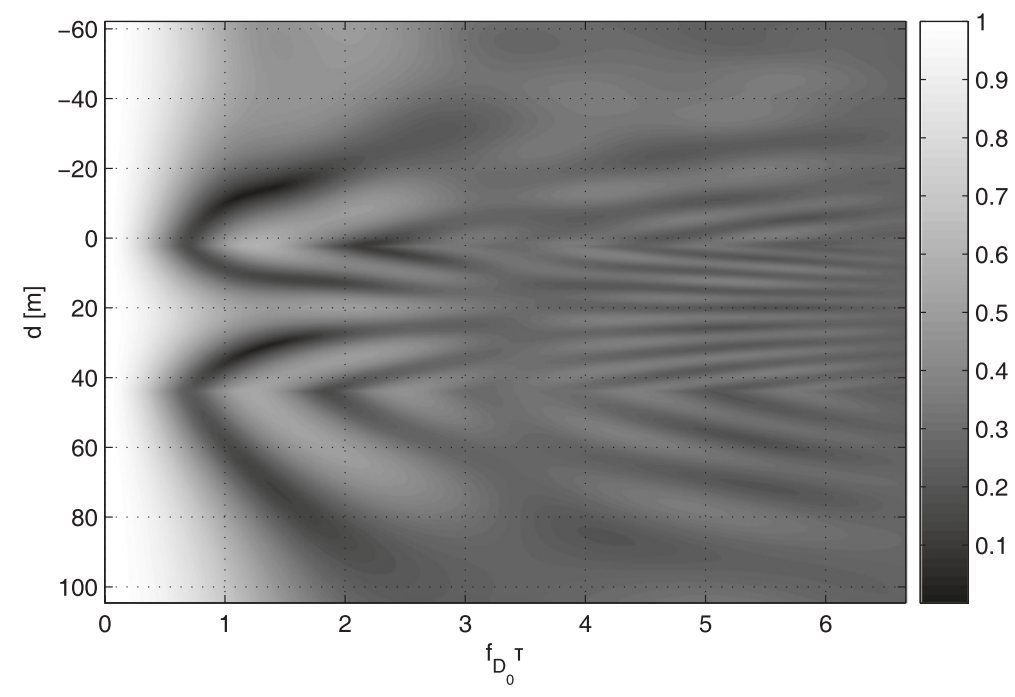

Figure 24 Auto-correlation function in a real-life scenario, Oxford Street - Wonderland Road intersection, London, ON, Canada.

cluster 2. As it is seen from the graph, cluster 1 almost does not contribute to the fading, cluster 2 and cluster 4 make the major contribution, and we may distinguish them on the auto-covariance function graph. Contribution of cluster 3 is merged with that of cluster 2, because this cluster is small and is located really close to the big cluster 2; therefore, the mobile is not able to differentiate between them. Effectively, it adds up to the power of cluster 2. Overall, the correlation snapshot appears blurred with a lot of grey levels corresponding to the correlation of 0.3 to 0.6 with no very pronounced dark areas (a very low correlation), as we saw in previous cases. The reason is that in this scenario, the distances between the mobile and clusters are bigger than in previous cases (30 to $64 \mathrm{~m}$ compared to 10 to $20 \mathrm{~m}$ ) as well as angular spreads $\left(25^{\circ}\right.$ to $35^{\circ}$ compared to $10^{\circ}$ to $\left.19^{\circ}\right)$. An example of MMSE and BER for 200 blocks frame-length and with three pilot signals prediction at $50 \mathrm{kbps}$ is shown in Figure 25, where we can see the influence of clusters at 0 and around $40 \mathrm{~m}$ (with respect to the second cluster).

In a similar way, the communication in any type of terrain, containing multiple obstacles, can be analyzed. Of course, extension to more complicated scenarios describing bigger number of clusters with non-symmetrical allocation is straightforward. Also, another various kinds of modulation and transmission schemes could be evaluated to improve the overall performance of the system.

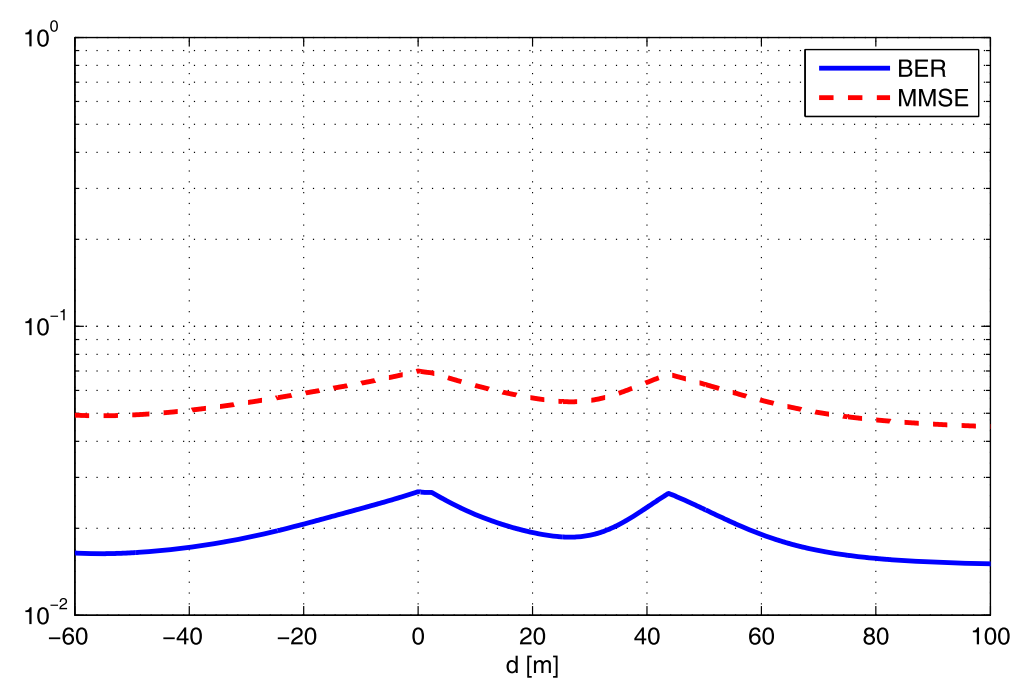

Figure 25 MMSE and BER as functions of distance to the cluster, Oxford Street - Wonderland Road intersection, London, ON, Canada. 


\section{Conclusion}

In this paper, MDPSS-based channel model was adopted for representing a practical environment containing one or more clusters whose geometry is known and predefined. It mimics realistic channels with non-symmetric spectra and complex-valued auto-covariance function, what allowed us to obtain more reasonable results. STTD communication system with Alamouti coding and pilotbased channel estimation was described in detail and applied to two different realistic scenarios: one of them depicts V2I communication with a mobile moving under a big cluster located on the way of the mobile, like a road sign. The other one sketched V2V case with two similar clusters located on one side of the road and two communicating mobiles passing by. The analysis of estimation quality were performed for each scenario. In both cases, an increase in estimation MMSE was detected in the vicinity of clusters resulting in the degradation of system performance in terms of BER. The effect of performance downgrading is larger in cases of longer frames between pilot signals, as a straightforward result from quickly decaying auto-covariance function of channel gains in occurrence of clusters in the environment. In the first scenario, the increase in MMSE and BER was higher than in the second scenario, although with shorter duration. Finally, an example of implementation of aforementioned channel model in simulation of communication at a real-life intersection was presented and discussed. It is worth mentioning that due to flexibility of the MDPSS simulator, the description of a vast variety of different scenarios is available, allowing one to easily test any kind of environment with different positioning of clusters in both V2V and V2I cases.

\section{Competing interests}

The authors declare that they have no competing interests.

\section{Acknowledgements}

The authors are supported by NSERC Canada.

Received: 30 January 2014 Accepted: 16 June 2014

Published: 11 August 2014

\section{References}

1. JK Cavers, An analysis of pilot symbol assisted modulation for rayleigh fading channels. IEEE Trans. Veh. Tech. 40, 686-693 (1991)

2. K Almustafa, S Primak, T Willink, K Baddour, On Achievable Data Rates and Optimal Power Allocation in Fading Channels with Imperfect Channel State Information, in IEEE 4th International Symposium on Wireless Communication Systems (Trondheim, Norway, 17-19 Oct. 2007)

3. J Jootar, JR Zeidler, JG Proakis, Performance of Alamouti space-time code in time-varying channels with noisy channel estimates, in IEEE Wireless Communications and Networking Conference (New Orleans, LA, USA, 13-17 March 2005)

4. D Tse, P Viswanath, Fundamentals of Wireless Communication (Cambridge University Press, New York, 2005)

5. H Zhu, B Xia, Z Tan, Performance analysis of Alamouti transmit diversity with QAM in imperfect channel estimation. IEEE J. Selected Areas Commun. 29, 1242-1248 (2011)

6. Z Tang, RC Cannizzaro, G Leus, P Banelli, Pilot-assisted time-varying channel estimation for OFDM systems. IEEE Trans. Sygnal Process. 55, 2226-2238 (2007)
7. J Andersen, N Blaustaein, Multipath Phenomena in Cellular Networks (Artech House, Norwood, 2002)

8. N Blaunstein, M Toeltsch, J Laurila, E Bonek, D Katz, P Vainikainen, N Tsouri, K Kalliola, H Laitinen, Signal power distribution in the Azimuth, elevation and time delay domains in urban environments for various elevations of base station antenna. IEEE Trans. Antenn. Propag. 54, 2902-2916 (2006)

9. MK Tsatsanis, GB Giannakis, Modeling and equalization of rapidly fading channels. Int. J. Adapt. Control Signal Process. 10, 159-176 (1996)

10. DK Borah, BD Hart, Frequecy-selective fading channel estimation with a polynomial time-varying channel model. IEEE Trans. Commn. 47, 862-873 (1999)

11. H van Trees, Detection, Estimation and Modulation Theory: Part 1, 1st edn. (Wiley, New York, 2001)

12. M Visintin, Karhunen-Loeve expansion of fast Rayleigh fading process IEEE Electron. Lett. 32, 1712-1713 (1996)

13. T Zemen, C Mecklenbrauker, Time-variant channel estimation using discrete prolate spheroidal sequences. IEEE Trans. Signal Process. 53, 3597-3607 (2005)

14. SJ Haghighi, S Primak, V Kontorovich, E Sejdic, Wireless communications and multitaper analysis: applications to channel modeling and estimation, in Mobile and Wireless Communications Physical Layer Development and Implementation (InTech Publishing, Rijeka, 2010)

15. C-X Wang, X Cheng, Vehicle-to-vehicle channel modeling and measurements: recent advances and future challenges. IEEE Commun. Mag. 47, 96-103 (2009)

16. J-H Lee, T Ernst, N Chilamkurti, Performance analysis of PMIPv6-based network mobility for intelligent transportation systems. IEEE Trans. Veh. Tech 61, 74-85 (2012)

17. S Haykin, Adaptive Filter Theory, 4th edn. (Prentice Hall, Upper Saddle River, 2001)

18. L Bernardo, A Roma, N Czink, A Paier, T Zemen, Cluster-based scatterer identification and characterization in vehicular channels, in 11th European Wireless Conference-Sustainable Wireless Technologies (European Wireless) (Vienna, Austria, 27-29 April 2011), pp. 1-6

doi:10.1186/1687-1499-2014-129

Cite this article as: Uchiteleva and Primak: Effect of scattering environment on estimation quality in V2I and V2V communications. EURASIP Journal on Wireless Communications and Networking 2014 2014:129.

\section{Submit your manuscript to a SpringerOpen ${ }^{\circ}$ journal and benefit from: \\ - Convenient online submission \\ - Rigorous peer review \\ - Immediate publication on acceptance \\ - Open access: articles freely available online \\ - High visibility within the field \\ - Retaining the copyright to your article \\ Submit your next manuscript at $>$ springeropen.com}

